\title{
Le pouvoir de la catégorie. Les politiques publiques et l'insertion professionnelle des immigrés en France et en Italie
}

The Power of Categories. Public Employment Policies and Immigration in France and Italy

El poder de las categorías. Políticas públicas e inserción laboral de inmigrantes en Francia y en Italia

\section{Annalisa Lendaro}

\section{OpenEdition}

\section{Journals}

Édition électronique

URL : https://journals.openedition.org/remi/5429

DOI : $10.4000 /$ remi.5429

ISSN : $1777-5418$

Éditeur

Université de Poitiers

Édition imprimée

Date de publication : 1 octobre 2011

Pagination : 35-55

ISBN : 979-10-90426-01-6

ISSN : 0765-0752

\section{Référence électronique}

Annalisa Lendaro, «Le pouvoir de la catégorie. Les politiques publiques et l'insertion professionnelle des immigrés en France et en Italie », Revue européenne des migrations internationales [En ligne], vol. 27 - $n^{\circ} 2$ | 2011, mis en ligne le 01 octobre 2014, consulté le 15 avril 2022. URL : http://

journals.openedition.org/remi/5429; DOI : https://doi.org/10.4000/remi.5429 


\section{Le pouvoir de la catégorie. Les politiques publiques et l'insertion professionnelle des immigrés en France et en Italie}

\section{Annalisa LENDARO ${ }^{1}$}

\section{INTRODUCTION}

Cet article propose une analyse de l'action publique via la reconstruction des processus d'insertion professionnelle des immigrés ${ }^{2}$. Il se propose d'expliciter les imbrications entre les parcours biographiques et les contextes politico-normatifs dans lesquels ils se déroulent.

L'intérêt est de mettre en lumière l'importance du contexte institutionnel (politiques d'emploi et d'immigration ; configurations locales d'acteurs) en termes d'opportunités d'accès à l'emploi et aux droits sociaux.

L'État contemporain et ses multiples traductions territoriales et/ou sectorielles essaient par différents instruments (Lascoumes et Le Galès, 2005) de modeler les tran-

1 Sociologue, Laboratoire d'Économie et Sociologie du travail, Université Aix-Marseille ; annalisa. lendaro@univmed.fr

2 En France, « immigré » est un terme utilisé en tant que catégorie statistique officielle. Sa définition a été fixée par le Haut Conseil à l'Intégration en 1990, mais elle n'a pas de valeur légale. En l'utilisant on fait référence aux personnes résidant en France depuis au moins un an et nées étrangères à l'étranger (elles peuvent avoir acquis la nationalité française). En revanche, «étranger » est une catégorie légale, et se réfère à toute personne qui n'a pas la nationalité française. En Italie, la catégorie légale est celle d' « étranger », mais on retrouve facilement le terme d' « immigré » dans les textes officiels. La tendance générale des politiques publiques, ainsi que pour le sens commun, est celle d'une équivalence presque parfaite entre ces deux catégories. Nous voulons souligner l'existence parallèle, en France comme en Italie, de classifications informelles d' " étranger ", qui opèrent le tri entre l'immigré communautaire et le non-communautaire, et également entre le non-communautaire « de luxe», auquel on ne se réfère pas avec le terme d'immigré (par exemple, Canadien) et le non-communautaire « de seconde classe » (par exemple, Maghrébin). 
sitions biographiques pour exercer son pouvoir sur les individus (Mayer et Schoepflin, 1989). Mais la question des influences entre action publique et parcours de vie devient depuis peu le véritable objet d'une production scientifique en science politique et en sociologie. L'étude des politiques sociales s'est naturellement prêtée à cet exercice (Giraud et Lucas, 2007).

Le parcours biographique a souvent été analysé comme si les différents évènements de la vie (Leclerc-Olive, 1997) s'enchaînaient dans un contexte a-politique ${ }^{3}$ : nous partons du postulat selon lequel l'action publique, par le biais de ses opérations de catégorisation des publics-cibles et de l'allocation différenciée de moyens, a un effet d'orientation sur les parcours de vie. Nous voulons montrer que l'entrée par les mécanismes d'insertion des immigrés est pertinente, car ces derniers représentent à leur tour un excellent analyseur de la société et de ses changements (Tripier, 2004).

Nous faisons l'hypothèse que les inégalités d'accès à l'emploi dépendent aujourd'hui de l'accessibilité de " statuts », qui renvoient à la position socioprofessionnelle, à l'appartenance générationnelle, à la légitimité et/ou à l'ancienneté de résidence sur un territoire, et aux divers niveaux de protection selon l'inclusion ou non dans les catégories-cibles de l'action publique. Non seulement ces dernières définissent des priorités de l'action publique, mais en outre affectent un statut juridique aux personnes (demandeur d'emploi, primo-arrivant, jeune sans qualification, etc.) qui ouvre droit à des services, à des allocations et à diverses autres formes de soutien; dans le même temps, elles excluent les non-éligibles. Les catégories-cibles sont également en mesure de définir des "life situations » (Mayer et Schoepflin, 1989), c'est-à-dire des périodes de la vie qui seraient caractérisées par des difficultés et des besoins typiques. Mais les parcours d'immigrés ici reconstruits feront ressortir des impasses, voire des paradoxes.

\section{ACTION PUBLIQUE ET LIFE COURSES : LES ENJEUX THÉO- RIQUES ET MÉTHODOLOGIQUES}

La démarche comparative nous est apparue comme un instrument précieux d'investigation (Lallement et Spurk, 2003), capable de faire émerger similitudes et différences dans la capacité des politiques publiques à orienter les parcours biographiques.

Une première échelle comparative se réfère à l'analyse de deux territoires régionaux, l'un en France (région Provence-Alpes-Côte d'Azur), l'autre en Italie (région Ligurie). La comparaison nous permet de considérer le contexte institutionnel et les catégories produites par l'action publique comme étant socialement situés (Dubar, 2001). Chaque contexte sociétal a sa propre manière de « construire » les catégories, les dispositifs, les politiques, les règles, le besoin en main-d'œuvre et la place des immigrés sur les marchés du travail.

L'approche comparative est complétée par un deuxième niveau qui s'attache à confronter deux types de parcours biographiques hétérogènes quant aux temporalités de

3 Nous rappelons néanmoins les travaux de Didier Demazière sur les chômeurs, de José Rose et de Chantal Nicole-Drancourt sur les jeunes. 
l'expérience migratoire pour chacun des deux contextes régionaux : il s'agit de comparer les modalités d'insertion professionnelle d'immigrés arrivés les uns dans les années 1980 et les autres au début des années 2000 .

Notre regard est donc tourné vers «les mécanismes méso qui constituent les liens entre individus et structures (institutions) »(Giraud, 2004 : 357). Les systèmes de visibilisation (Lendaro, 2010) de la main-d'œuvre immigrée, les modalités de gestion de l'information et les formes de coordination entre acteurs du marché sont appréhendés à partir de parcours biographiques. Ces derniers sont capables de rendre compte de l'imbrication de contraintes et d'opportunités qui relèvent à la fois de l'individu et de son contexte institutionnel ${ }^{4}$.

En France par exemple, les immigrés font rarement l'objet d'une intervention spécifique. À ce titre, nous soulignons l'intérêt d'une analyse critique des catégorisations retenues par l'action publique, afin de mettre en évidence la mesure dans laquelle les acteurs «visibilisent » les populations immigrées par le biais d'opérations de ciblage, qui ouvrent des possibilités, mais imposent également des choix et des contraintes aux individus.

Le détour par la situation italienne permet d'apporter un regard nouveau sur les politiques françaises de l'emploi et sur la gestion de la main-d'œuvre immigrée à travers un exercice comparatif qui s'inspire de l'analyse sociétale « revisitée $»^{5}$ (Verdier, 2000).

Comment l'action publique structure-t-elle les parcours ou du moins essaye-telle de réguler les transitions des individus sur le marché du travail ? Les immigrés sont-ils concernés par des «transitions spécifiques » qui les distinguent des autres citoyens ? Quel est dans ce cas le lien avec l'accès à un statut reconnu, comme par exemple celui de salarié, ou d'immigré régulier?

Notre démarche consiste à reconstruire les parcours biographiques en tant que produit d'une histoire individuelle de migration et d'une contextualisation de l'acteur, qui renvoie à son double ancrage, individuel et institutionnel.

En ce sens, la mise « en situation » que les acteurs font d'eux-mêmes à partir de la réflexion sur leur propre parcours nous aide à faire émerger non seulement le système de choix-contraintes-opportunités, mais aussi les "parcours-type » forgés par l'action publique, qui fixent les caractéristiques des transitions « standards ».

4 Le cas du bâtiment nous est apparu intéressant à plusieurs titres. Ce secteur est traditionnellement ouvert à l'emploi de la main-d'œuvre immigrée, et généralement présenté par la branche comme un secteur d'insertion en permanente demande (Jounin, 2008). Il est considéré «labour intensive », caractérisé par un emploi massif de personnel peu qualifié, mais physiquement très sollicité ; de ce fait, il n'est guère attractif pour la main-d'œuvre autochtone (Ive et Gruneberg, 2000), en raison également de taux élevés d'accidents et de maladies professionnelles. De plus, le bâtiment est un univers où coexistent des formes de stabilité d'emploi avec une diffuse incertitude contractuelle, caractérisé par l'éclatement du collectif de travail (Mashkova, 2009), du fait notamment de la hausse importante du recours à la main-d'œuvre intérimaire et à la sous-traitance (Fellini, Ferro et Fullin, 2007).

5 Cette approche introduit l'action publique dans l'analyse sociétale "classique » de Maurice, Sellier et Silvestre (1982) qui avaient montré l'interdépendance de plusieurs systèmes (éducatif, de relations industrielles, économique, etc.) dans le cadre des réajustements du marché du travail. 
L'analyse de quatre parcours, deux pour le cas français et deux pour le cas italien, met en scène des dynamiques sociales liées aux contextes institutionnels des deux territoires, en remettant en cause les catégories officielles qui laissent souvent transparaître une conception de la population en « stock » plutôt qu'en « flux ».

À travers quels mécanismes l'action publique contribue-t-elle à orienter les parcours de vie ? De quelle manière la visibilisation de l'immigré comme catégorie-cible fait-elle émerger des parcours-type et favorise ou inhibe les inégalités d'accès à l'emploi ?

Nous focaliserons notre attention sur des évènements perturbateurs (Grossetti, 2006) dans trois sphères de la vie : l'emploi, le statut administratif (régularisation et titre de séjour) et la situation familiale.

L'insertion professionnelle est donc tout à la fois historiquement inscrite dans une conjoncture politique, économique, migratoire. Le récit des individus nous parle de la manière dont le sujet s'approprie un contexte, en adoptant des stratégies, en opérant des choix « obligés » en construisant son parcours, qui est dans tous les cas lié au passage d'un statut à un autre. C'est dans ce passage que nous allons chercher ensuite à identifier le rôle de l'action publique.

\section{PARCOURS (ET TENTATIVES) D'INSERTION EN PROVENCE- ALPES-CÔTE D'AZUR (PACA)}

En PACA, comme partout en France, les politiques publiques (sociales, de l'emploi, etc.) ne prennent pas en compte des critères ethniques dans la distribution des ressources et des services. La politique de la ville, par exemple, a élaboré des formes d'intervention publique fondées sur le lieu de résidence. Ces mesures ont été définies à travers la formule de « discriminations territoriales positives » (Béhar, Estèbe et Epstein, 1998 : 86). L'approche territoriale est devenue un des instruments pour intervenir sur des problématiques dont la dimension ethnique est en arrière-plan (Doytcheva, 2007), en présentant les démarches comme des actions généralistes en faveur des résidents de la géographie « exclusive » de la ville.

Dans le domaine de l'emploi, l'intégration des travailleurs immigrés ${ }^{6}$ a longtemps été un produit joint de l'appel utilitariste à cette main-d'œuvre (Viet, 1998 ; Tripier, 1991). L'accès à l'emploi était porteur d'une double reconnaissance : l'acquisition d'un statut professionnel et l'accumulation d'un capital humain par le biais d'une expérience professionnelle reconnue en tant qu'ancienneté. Le rationnement de l'emploi a pourtant aiguisé la compétition entre les outsiders et pérennisé l'enclavement de certains d'entre eux à la

$6 \mathrm{Au}$ total, cette intégration par l'emploi (Castel, 1995) et la protection sociale qui lui était attachée ont certes toujours moins bien fonctionné à l'endroit des personnes immigrées (Linhart, 1978 ; Beaud et Pialoux, 1998), mais il reste que la stabilisation dans l'emploi permettait néanmoins à beaucoup de ces travailleurs d'accéder au fil du temps aux principaux attributs de la citoyenneté sociale. 
périphérie des marchés internes, les immigrés au premier chef ${ }^{7}$.

Les politiques publiques françaises se sont attachées à cibler des groupes spécifiques (jeunes, handicapés, chômeurs, RMIstes, etc.) tout en excluant de prendre en considération les différences ethniques et ce, dans le but d'assurer l'égalité des chances. Le statut d'immigré n'est pas reconnu en tant que catégorie de l'action publique concernant le marché du travail, parce que déjà inclus dans toutes les catégories-cibles utilisées ; en quelque sorte, elle est en arrière-plan, invisible parce qu' " intégrée » aux autres catégories.

Plus récemment, les instruments de l'action publique, notamment dans le domaine de l'emploi et de la formation, reflètent une ambition : faire en sorte que les politiques agissent sur le parcours de l'individu, de manière à construire un cheminement d'accompagnement à l'emploi constitué par différentes options (formation, contrats aidés, reconversion, etc.) qui offriraient une solution plurielle, en mesure d'éviter que l'individu se retrouve dans une situation de « non-droit».

Comment se décline cette notion de parcours lorsqu'il s'agit de considérer l'accompagnement à l'emploi et à la formation de l'immigré, étranger notamment ? Les politiques publiques prennent-elles en considération les besoins de l'individu au point de s'interroger sur les exigences spécifiques de l'étranger non-communautaire ? Comment ce questionnement émerge-t-il à partir du récit des protagonistes immigrés ?

Le premier parcours introduit cet enjeu en montrant comment la stabilité de l'emploi est liée à l'acquisition du titre de séjour et à l'accès à un emploi déclaré.

\section{Ali (Comores/Marseille) : la stabilité et la contrainte}

Ali est Comorien, il a trente-huit ans et a quitté son pays en 1988. Lors de notre rencontre, il était maçon dans un chantier à Aix-en-Provence. Il a abandonné l'école en seconde pour " tenter sa chance » en France. Une fois à Marseille il a "bricolé », il a commencé à aider un copain sur un chantier, et puis il a connu son actuel patron. Ali travaille avec lui depuis vingt ans ; quand son patron s'est mis à son compte, Ali a été embauché en CDI (1995). Depuis 1996 il a une carte de séjour.

Ali habite depuis toujours à la Belle de Mai, un quartier populaire de Marseille, où la communauté comorienne est nombreuse. Il n'est pas marié, mais il a « une femme » et plusieurs enfants, nés en France. Aux Comores il a encore de la famille, et notamment sa mère, à laquelle il envoie régulièrement de l'argent pour lui assurer une vieillesse sereine.

Le personnage clé qui émerge du récit d'Ali est sans doute son employeur. Ali y revient continuellement en faisant aussi référence à l'événement clé qui lui est associé, c'est-à-dire l'obtention du CDI en 1995. Cet événement a un poids considérable sur toutes les sphères de sa vie, sur son projet migratoire et sur ses familles, « ici » et « là-bas ». Certes, quand il est arrivé à Marseille les amis comoriens l'ont « dépanné » en l'héber-

7 Les emplois précaires et les situations de « manque d'un statut », aujourd'hui comme avant, sont plus fréquents parmi les jeunes, les femmes, les non-diplômés, les immigrés, ces derniers réunissant souvent plusieurs des conditions précédentes (Piore, 1979 ; Tripier, 1990). 
geant chez eux et en le mettant en contact avec cet artisan qui l'a fait « bricoler ». Mais c'est son patron qui lui a permis de " gagner le pain », d' " apprendre sur le tas » et de « monter l'échelle ». C'est grâce à ce patron qu'Ali a pu obtenir son titre de séjour provisoire et ensuite sa carte de séjour, et c'est aussi par la confiance qu'il lui a accordée qu'il est devenu maçon. Ali associe à son patron la possibilité de rester en France parce qu' « il voulait me garder » et parce que « tu sais... pour résoudre tes problèmes administratifs il faut avoir quelqu'un de ton côté ».

Dans le récit d'Ali, tout ce qui est de l'ordre de la contrainte concerne les papiers et sa famille : celle d'ici (les enfants) et celle de là-bas (sa mère). Ce triptyque correspond à l'ensemble des éléments qui entrent en jeu lorsqu'Ali me parle de ses projets impossibles à réaliser (le retour au pays), des contraintes qu'il subit quotidiennement (« aller travailler tous les jours pour pas grand-chose », « nourrir les enfants », « envoyer l'argent au pays »), et de la possibilité de se tenir au moins à l'obligation morale d'aider sa mère aux Comores.

L'accès au statut d'immigré régulier avec permis de travailler a fait que son objectif prioritaire a été réalisé, c'est-à-dire rester en France et gagner de l'argent à envoyer à sa famille aux Comores. Ali ne me parle pas beaucoup de l'administration française, et il se limite à répéter que « c'était très difficile », mais que grâce à son patron il a pu « devenir quelqu'un qui a le droit d'être ici ». Le permis de séjour signifie donc à la fois se sentir légitimé à rester sur le territoire français et à le faire sans être victime de remords vis-à-vis de sa mère restée au pays. Cependant ce statut, lié à l'activité dans le bâtiment qu'il dit lui rapporter peu d'argent, l'oblige à rester en France alors que son projet initial était de « travailler pour revenir au pays, parce qu'il n’y a pas mieux que chez soi ». Ce projet a été abandonné : Ali a eu des enfants en France et « quand t'as des enfants c'est pas pareil, tu es condamné ».

De plus le fait de se sentir légitimé à rester, lié au statut d' " immigré régulier », ne suffit pas à se sentir « comme les autres» : il se décrit comme un "étranger », parce que « pour les autres t'es toujours un étranger». Encore une fois c'est le patron qui a un rôle déterminant puisqu'Ali précise tout de même que « tant que ton patron te considère comme tout le monde, ça va ».

Patron et papiers deviennent donc les éléments qui font de son expérience un parcours d'insertion («j'ai quand même la stabilité de l'emploi »), même si « la maçonnerie c'est pénible et mal payé, c'est pour ça qu'on y retrouve que des Noirs et des Arabes ».

Son « monde » (Dubar et Demazière, 1997) est donc celui de la « contrainte stabilisée ", où l'État prend la forme de l'institution qui formalise son acceptabilité en tant qu'étranger avec des droits de séjour, qui passe par l'appui de son patron, vrai décideur politique informel.

L'accès à ce statut s'est produit pour Ali dans un contexte politique particulier. La politique migratoire française et les formes de régulation du marché du travail ont évolué, en modifiant la marge de manœuvre des immigrés arrivés plus récemment en France, comme dans le cas d'Abdel. 


\section{Abdel (Maroc/Aix-en-Provence) : l'incertain et l'accès à un quasi- statut}

J'ai connu Abdel en 2006. Il est Marocain d'un quartier populaire de Casablanca et il est arrivé en France en 2005 avec un visa « étudiant». À son arrivée, il avait vingtsept ans et venait d'avoir sa licence en économie à l'Université de Casablanca. Il avait demandé un visa pour s'inscrire en maîtrise dans une université française. En 2005, il s'est inscrit à l'Université de Montpellier et en 2006 il a obtenu son diplôme. Cette époque est pour lui : « le seul moment depuis que je suis en France, que je n'ai pas été en galère et que j'avais espoir de faire quelque chose de bien ».

«Quelque chose de bien » pour Abdel signifie trouver un emploi, car son objectif en venant en France a toujours été de «travailler, gagner ma vie ». Les études ne l'intéressent pas vraiment puisque le visa « étudiant» pour lui n'est qu'un permis de résidence. Abdel, en effet, comptait terminer son cycle universitaire en France, " avec un diplôme français, parce que nous (les immigrés) on peut avoir le diplôme le plus élevé et prestigieux qu'on connaît, mais s'il n'est pas français, ça compte que dalle », pour ensuite chercher un emploi en France, « dans une entreprise, dans la logistique ».

La première année en France se déroule « normalement » : «c'était normal, je bossais mes cours, j'avais un visa étudiant et j'avais réussi à avoir une chambre en cité universitaire comme les autres étudiants, c'était nickel ». Pour se faire un peu d'argent de poche, Abdel a aussi travaillé quelques week-ends au black, dans un restaurant du centreville comme plongeur et aide-cuisinier.

Le tournant est la transition entre l'obtention de son diplôme de maîtrise et la recherche d'un travail ou d'un stage dans le domaine visé (la logistique). Il commence ses recherches, sans succès : «Je cherchais partout, même des stages non rémunérés. Personne ne m'a contacté $»$.

Entre-temps son visa expire, et il doit trouver rapidement une solution de « dépannage » : il ne voit que la possibilité de s'inscrire une deuxième fois à l'université, pour renouveler son titre de séjour. Mais Abdel veut « se faire des sous, maintenant j'ai assez étudié et ma famille attend que je leur envoie de l'argent ». Finalement, faute de mieux, il s'inscrit une deuxième fois en master 1, cette fois à l'Université d'Aix-enProvence. Mais les problèmes ne font que commencer. D'abord pour l'inscription : il a fallu convaincre des compatriotes de Montpellier, étudiants à leur tour, de lui prêter l'argent nécessaire. Ensuite pour le logement; en 2006 Abdel vient d'avoir vingt-huit ans et il n'est plus éligible pour l'attribution d'une chambre en cité universitaire : « la dame du CROUS... elle ne veut rien entendre, elle me dit que je n'ai pas la priorité, qu'il faut que je cherche dans le privé ! ». Cela pose des problèmes au niveau de son permis de séjour car il faut qu'il trouve rapidement quelqu'un pour lui faire une attestation d'hébergement. Et sur Aix-en-Provence il ne connaît personne, donc c'est toujours auprès de ses amis de Montpellier, et en particulier de sa copine française, qu'il arrive à « gratter l'attestation ».

Entre le moment où il dépose (avec un mois et demi de retard) son dossier de renouvellement et le moment du retrait du visa à la sous-préfecture d'Aix, huit mois 
passent. « Huit mois de galère », selon Abdel. L'angoisse pour le titre de séjour est récurrente dans son récit et la sous-préfecture devient un protagoniste omniprésent : « là-bas (à la sous-préfecture), ils n'attendent que de me renvoyer chez moi ! ». Il cherche ainsi « d'autres types d'investissement». Le premier qu'il évoque est le PACS : il me dit avoir commencé à mettre la pression à sa copine de Montpellier, pour qu'ils se pacsent parce que « tu sais, pour un immigré, quand t'es pacsé avec une Française, après ton dossier passe mieux, et t'as aussi plus de chances de trouver un boulot ». Le deuxième est un effort d'élargissement de son réseau : " je dois me faire des amis qui puissent me filer du taf ». Le « taf » en question devient la vente du « shit». Il m'en parle sans honte, mais en adoptant toujours le registre de la contrainte, en m'expliquant qu'il fallait trouver une source de revenus.

Abdel se trouve face à un moment critique : ses compatriotes qui l'hébergent à la cité universitaire d'Aix lui font comprendre qu'ils ne sont pas en situation de prendre des risques à cause de ses trafics ; entre-temps sa copine décide de le quitter.

C'est dans ce contexte (juillet 2006) qu'un autre personnage apparaît, qui joue un rôle déterminant dans son parcours. Il s'agit d'un « cousin » marocain : Mohammed. Il travaille comme maçon dans le bâtiment et propose à Abdel de l'aider de temps à autre sur les chantiers quand son patron en a besoin. C'est ainsi qu'Abdel découvre le secteur du bâtiment, en travaillant ponctuellement comme manœuvre au black. La vie de chantier lui permet de découvrir les possibilités d'emploi que le bâtiment pourrait lui offrir et surtout il commence à se faire une idée des statuts des travailleurs qu'on peut rencontrer dans ce secteur : « les intérimaires, eux, c'est mieux. Ils gagnent leur vie, après s'ils sont fatigués ils prennent des vacances, vont voir la famille... ». C'est ainsi qu'Abdel prend le prétexte d'une embrouille avec le patron (« il ne m'a pas payé tout ce qu'il me devait, je ne suis pas son esclave non plus ! ») pour partir, et il décide de chercher des missions dans les agences d'intérim.

Mais tout ne se passe pas comme il le souhaite : d'abord, il n'a pas encore retiré son visa, il a juste le récépissé sept mois après la date de dépôt du dossier. Ensuite, il est étudiant, donc formellement autorisé à travailler dans la limite de $60 \%$ de la durée de travail annuelle et l'employeur qui souhaite l'embaucher ne peut le faire qu'après déclaration nominative effectuée auprès de l'administration (sous-préfecture et DDTEFP). Abdel est donc censé obtenir une autorisation provisoire de travail ${ }^{8}$. De plus, un document essentiel nécessaire aux entreprises de travail temporaire est le justificatif de domicile de moins de trois mois, qui fait qu'Abdel est obligé de trouver quelqu'un qui certifie de sa résidence à l'extérieur de la cité universitaire ${ }^{9}$. Abdel entre en contact avec deux agences d'intérim d'Aix spécialisées dans le bâtiment, mais à chaque fois il se démotive face aux contraintes administratives. Il prend donc du retard dans l'envoi des documents demandés par l'agence et il finit par se désister.

Quand je rencontre Abdel pour la première fois, il est dans cette phase de démoti-

8 Art. 9 de la loi $\mathrm{n}^{\circ}$ 2006-911 du 24 juillet 2006. Depuis le $1^{\text {er }}$ juillet 2007, l'autorisation provisoire de travail pour les étudiants étrangers n'est plus obligatoire.

9 Légalement, il n'est pas possible de se faire héberger, car la taille des chambres est de neuf mètres carrés. 
vation, et ses projets originaux sont en train de s'écrouler. Il porte un regard très amer sur sa vie en France et il a la sensation d'être traqué : « Maintenant, pour le CROUS je suis trop vieux, je n'ai plus les droits d'un étudiant, même si je suis inscrit à la fac. Mais pour les autres (la sous-préfecture, la DDTEFP), je suis un étudiant et pas un vrai travailleur ! C'est galère ». La désillusion ressort constamment de son récit : le visa qu'il récupère en août 2006 est un visa " étudiant » et Abdel commence à se rendre compte de la difficulté qu'il aura à renouveler son permis de séjour et à le reconvertir en titre " de travail » : «Moi, la fac c'était juste pour les papiers, moi je suis venu pour bosser, mais... passer d' " étudiant étranger » à " travailleur étranger » ça va être difficile... ». L'angoisse est accentuée par les temps administratifs assez contraignants : en août, Abdel retire son visa, qui expire en octobre de la même année. À partir de ce moment, je n'ai plus rencontré Abdel.

Abdel est le détenteur d'un « quasi-statut » : étranger autorisé à résider en France parce qu'étudiant, il aspire à devenir un travailleur. Il est toutefois doublement illégitime (en tant qu'étudiant parce que « non plus jeune » et en tant que salarié parce qu'étudiant), donc dépendant des réseaux et des opportunités informelles du marché local.

\section{POLITIQUES PUBLIQUES ET INSERTION DES IMMIGRÉS EN ITALIE : UN DÉTOUR PAR LA LIGURIE}

Le cas italien, analysé dans le cadre d'une comparaison avec la France, est particulièrement révélateur de l'importance du contexte politico-normatif.

Ce qui caractérise l'Italie est la rapidité du passage de pays d'émigration à celui de pays d'immigration. Cette transformation s'est opérée de manière spontanée et quasiment pas réglementée à partir de la deuxième moitié des années 1980 : dans une première phase, l'immigration s'est développée " par le bas », au sein du marché du travail formel et informel, et ce n'est que dans un deuxième temps que ce phénomène a été reconnu par les institutions publiques à travers un corpus normatif $a d$ hoc.

Dans un premier temps, la prise de conscience publique concernant ce changement se limite à une vision assez négative présentée très sommairement comme un nouveau problème social (Ambrosini, 2008) parmi bien d'autres qui marquaient l'Italie des années 1990.

Mais les acteurs du marché du travail (entreprises, syndicats de salariés, patronat, etc.) et la société civile (associations, groupes religieux et caritatifs, etc.) ont commencé à en « réguler» le fonctionnement par le biais d'arrangements, informels et fragmentés, bien avant la prise en charge par l'État de la gestion de la présence immigrée sur le territoire (Zincone et Caponio, 2004). Dans ce contexte, la catégorie d'immigré n'a pas donné lieu à des débats éthiques et conceptuels comparables à ceux qui font encore la une de l'actualité politique et scientifique en France. 
Les solutions politiques et les formes de régulation étatique ont suivi un mouvement déjà déclenché par le marché, et les lois de régularisation ${ }^{10}$ témoignent d'un dispositif d'immigration caractérisé par une continuité de l'approche « action dans l'urgence » (Barbagli, Colombo et Sciortino, 2004). L'Italie n'a pas encore adopté un système normatif qui permet d'intervenir sur l'insertion professionnelle des immigrés, sans que cela ne se fasse a posteriori pour résoudre des « urgences sociales » (travail informel, accidents sur le lieu de travail, etc.). Cela nous aide à comprendre pourquoi en Italie, lorsque les immigrés nous livrent leurs parcours de vie, il est tout à fait normal de passer par une phase de non-statut et de vivre la condition de « sans-papier » comme une période difficile, mais transitoire.

C'est une forme de bricolage territorial qui œuvre en amont des dispositifs institutionnels de régulation et d'insertion professionnelle des immigrés et qui parfois anticipe les interventions étatiques, par le biais de pratiques informelles dans l'accès aux droits sociaux (dans le cadre de la formation professionnelle, de l'activité syndicale, etc.). Le contexte sociétal est donc un espace pertinent d'analyse des processus d'insertion des immigrés et certains acteurs jouent un rôle clé dans la construction sociale de la rencontre entre la demande et l'offre de travail (Ambrosini, 2001 et 2005). Parmi ces acteurs, nous avons retrouvé, dans le récit de nos deux travailleurs immigrés à Gênes, les entrepreneurs, les réseaux (ethniques ou non) et les agents de la société civile et de la solidarité (syndicats de salariés, associations, groupes de bénévoles, paroisses, etc.) ; tous, d'une manière ou d'une autre, ont participé à l'orientation de ces parcours de vie, ainsi qu'à la structuration progressive de la réponse publique au phénomène migratoire, en intervenant précocement sur l'accueil des immigrés et sur la nécessité de mettre en place un système de régularisation, tout en suscitant et amplifiant les résistances xénophobes d'une partie de la société italienne.

\section{Karim (Tunisie/Gênes) : de la clandestinité à la sécurité professionnelle}

Entre octobre et décembre 2008 j'ai accompagné un syndicaliste de la FenealUIL ${ }^{11}$ lors de l'une de ses tournées dans le département génois, dans le but de rencontrer ses adhérents inscrits et de susciter de nouvelles adhésions au syndicat. Ainsi, j'ai rencontré Karim à l'occasion d'une visite sur un chantier. Karim est Tunisien. Il a quarante-trois ans, et séjourne en toute régularité à Gênes, car il a obtenu la nationalité italienne en 1992 suite au mariage avec son ex-femme italienne. Mais cet évènement a clôturé un parcours fort accidenté.

Il était parti de Tunisie en 1988 à vingt-deux ans. À cette époque, il était relativement facile de rentrer en Italie car l'État italien ne demandait pas de visa : pour avoir un permis de séjour de trois mois pour tourisme, il suffisait de pouvoir justifier d'un niveau minimal de ressources, plus ou moins équivalent à 150 euros. Sa famille a appuyé sa démarche, ses parents ont collecté l'argent nécessaire et Karim est donc parti accompagné

10 La première vraie « sanatoria » a lieu en 1990 avec la loi dite « Martelli ». Cinq lois de régularisation en vingt ans suivent.

11 Federazione Italiana Lavoratori Edili Affini e del Legno, le syndicat du BTP de l'Union Italienne des Travailleurs (UIL). 
d'un ami. Ils ont débarqué à Gênes et ils sont restés une semaine dans un petit hôtel. Après leur séparation à cause d'une dispute, Karim s'est retrouvé à Gênes, sans connaître personne, sans logement et avec son passeport timbré qui lui permettait de séjourner régulièrement sur le territoire au plus trois mois, et sans autorisation de travail.

Deux semaines après son arrivée, il trouve un petit boulot dans une ruelle du centre historique : au black, il empaille des chaises. Karim avait déjà fait ce travail en Tunisie quand il était jeune. C'est en s'arrêtant devant la vitrine d'un atelier qu'il s'est fait embaucher : le patron voulait le chasser, inquiété par son aspect négligé. Karim, qui ne parlait pas l'italien, lui fait comprendre qu'il connaissait le métier et le patron, surpris, lui propose de travailler avec lui. Karim précise de suite qu'il n'a pas de papiers, et le patron le rassure avec un éloquent « encore mieux ! ». Karim se montre volontaire et le patron lui propose de rester six mois. N'ayant pas de permis de travail, le patron le rémunère symboliquement en le logeant dans une petite chambre et en le nourrissant.

La transition se fait par le biais d'une fille italienne, qui se propose de le loger, et avec laquelle il instaure une relation amoureuse. Elle présente Karim à un artisan du bâtiment qui lui offre une première expérience dans le secteur. Karim travaille avec lui quatre mois comme manœuvre au black, puis l'artisan lui dit qu'il n'a plus besoin de lui. Quelques semaines après, sa copine le quitte et lui dit de se chercher un logement ailleurs.

À partir de ce moment, Karim me dit avoir passé une longue période de « grosse galère ». Sans travail ni permis de travail, sans logement et sans amis ou famille sur place, il ne pouvait compter que sur lui-même. Il résiste presqu'un an en dormant entre les dortoirs publics de la Caritas ${ }^{12}$ et la plage, en se protégeant derrière les petits bateaux des pêcheurs. Il me dit avoir pensé plusieurs fois rentrer en Tunisie, avoir été affamé, avoir craint de se déplacer à pied ou avec les moyens de transport public à cause des contrôles.

En 1990, l'Italie met en place la première grande régularisation de sans-papier ${ }^{13}$, grâce à la loi dite « Martelli » ${ }^{14}$, du nom du ministre socialiste de la justice (février 1991février 1993). Ainsi Karim, qui avait été informé par un Marocain qu'il avait connu dans un dortoir, décide de se présenter à la préfecture de Gênes pour entamer la procédure de régularisation. Mais le chargé d'informations répond à la demande de Karim en lui disant qu'il n'a pas le droit de bénéficier de cette opportunité de régularisation, puisque la loi concerne les étrangers entrés sur le territoire avant une certaine date, et cela ne serait pas son cas. Or, Karim rentrait précisément dans les cas prévus par la loi Martelli : « je ne sais pas pourquoi ce monsieur m'a dit que ce n'était pas possible, tu perds la raison dans ces situations comme ça... !».

Pourtant, l'évènement qui change sa vie ne tarde pas à arriver : Karim rencontre par hasard un syndicaliste de la CGIL $^{15}$. Après avoir écouté son histoire, il l'invite à passer

12 Association caritative de solidarité liée à l'évêché de Gênes et à l'Église catholique.

$13 \mathrm{Au}$ final, 200000 étrangers sont régularisés en 1990, majoritairement originaires des pays du Maghreb.

14 Loi n 39 du 28 février 1990, qui traduit le décret n 416 du 30 décembre 1989.

15 Confederazione Generale Italiana del Lavoro. 
au siège départemental de la Fillea ${ }^{16}$, afin de préparer le dossier de régularisation. Karim suit son conseil et il dépose son dossier à la préfecture deux semaines après. Entre-temps, ce même syndicaliste suggère à Karim d'aller s'inscrire au cours de formation pour maçon de l'École du bâtiment ${ }^{17}$. Karim se présente avec le récépissé du dépôt de demande de régularisation, mais la secrétaire de l'École lui répond qu'il ne sera pas possible de s'inscrire tant qu'il ne pourra exhiber son titre de séjour. Karim revient donc au syndicat, où le syndicaliste le pousse à insister et à rappeler l'École du bâtiment afin de prouver de son intérêt pour le cours. Au troisième appel téléphonique, la secrétaire l'accepte et l'inscrit muni du seul récépissé, en lui expliquant que l'École n'avait pas atteint le nombre minimum pour faire démarrer le cours et que donc la direction avait décidé de « faire des exceptions ».

Le cours suivi par Karim dure six mois, pendant lesquels il reçoit une modeste indemnité financière (180 euros par mois). Il est hébergé et nourri, tout comme les autres stagiaires, dans un couvent. C'est à l'École du bâtiment que Karim connaît celle qui deviendra sa femme : il s'agit de la secrétaire de l'école.

Karim est régularisé et entame la procédure pour avoir la nationalité italienne, termine son cours de maçon et trouve, par l'intermédiaire de l'École du bâtiment, d'abord un stage dans une entreprise, puis un CDD en tant qu'ouvrier spécialisé ( $3^{\text {ème }}$ niveau), grâce à sa femme qui s'occupe des contacts avec les employeurs. Au bout de huit mois, cette entreprise fait faillite et Karim trouve un CDI dans une troisième entreprise, où il ne reste que peu de temps à cause des mauvais rapports avec son chef. La quatrième entreprise qui l'embauche, toujours en CDI et en tant que maçon, est celle pour laquelle il travaille depuis seize ans.

Le parcours de Karim témoigne d'une réussite. Le rôle joué par les acteurs du marché du travail (syndicat, École du bâtiment) est fondamental, mais met en évidence aussi les arrangements possibles au niveau local.

\section{Ricardo (Équateur-Gênes) : non-insertion et manque de statut}

Ricardo est Péruvien, il est arrivé en Italie en 2005 alors âgé de vingt-cinq ans, avec un faux passeport argentin. Père d'un enfant atteint d'une malformation cardiaque, il est parti en 2003 du Pérou d'abord vers l'Argentine à la recherche d'un travail dans le pays économiquement plus prospère de l'Amérique du Sud.

En Argentine, Ricardo trouve de temps à autre du travail non déclaré dans le bâtiment, mais cela représente des entrées économiquement maigres et discontinues. Il commence donc à réfléchir au moyen de partir en Italie, car il a un ami péruvien qui s'est installé à Rome et une cousine qui est partie deux ans auparavant à Gênes. À Buenos Aires, Ricardo fait la connaissance d'un garçon qui travaille dans un bureau d'État Civil. Après paiement d'une somme conséquente, cet agent lui délivre un faux acte de naissance

16 Federazione Italiana Lavoratori del Legno, Edili e Affini.

17 Organisme paritaire de branche qui en Italie existe dans chaque département et qui est chargé de mettre en place les formations professionnelles concernant les métiers du bâtiment. 
qui lui servira pour demander son passeport argentin pour entrer en Italie ; des ressortissants de ce pays, l'Italie n'exige pas de visa.

Ricardo obtient son passeport et s'endette pour acheter son billet d'avion pour Rome. Angoissé par le risque d'être arrêté par la police de l'air, il part en 2005, après la mort du Pape Jean-Paul II, faisant en sorte que son déplacement passe pour un voyage d'ordre religieux. Arrivé à Rome, Ricardo retrouve son " ami », qui disparaît après lui avoir fait miroiter un hébergement et la possibilité d'un travail. Ricardo se met alors en contact avec sa cousine qui l'invite à la rejoindre à Gênes.

Ricardo arrive en Ligurie et s'installe chez sa cousine ${ }^{18}$, qui travaille en tant qu'aide à domicile. Il reste presque un mois sans travailler, sans pouvoir compter sur le réseau de sa cousine pour la recherche d'un emploi ${ }^{19}$. Enfin, il décide de demander conseil auprès de la paroisse que sa cousine fréquente. C'est là un point de référence des immigrés sud-américains à Gênes, où « les prêtres et les sœurs nous aident à trouver du travail ou à trouver un logement quand on est en difficulté ». Au prétexte d'un cours d'italien gratuit organisé par la paroisse et ses bénévoles, Ricardo commence à fréquenter ce milieu. Il rencontre d'autres sans-papier et demande de l'aide dans la recherche d'un travail : un Péruvien lui trouve du travail au noir dans le bâtiment. Il reste cinq mois chez un artisan, qui le paye très irrégulièrement, en travaillant entre dix et douze heures par jour. Au bout de trois mois, Ricardo voit son dos se bloquer. À l'hôpital le médecin, qui diagnostique une déchirure musculaire, lui conseille de rester au repos deux semaines. Mais après cinq jours Ricardo retourne au chantier : il avait besoin d'argent et craignait que son patron ne le remplaçât par quelqu'un d'autre. Et les choses se passent mal : non seulement à cause des douleurs que Ricardo ressent encore lors de notre entretien, trois ans après l'accident, mais aussi parce que son patron le « licencie », sans lui régler les deux derniers mois de salaire.

Ricardo est désespéré et commence à exercer des pressions sur son patron afin d'être payé, en lui expliquant sa situation de sans-papier et celle de sa famille au Pérou. Alors que son patron ne répond plus au téléphone après lui avoir promis son dû, Ricardo décide de se présenter sur le chantier. Son patron lui donne alors un rendez-vous à son domicile, pour régler l'affaire. Le jour suivant, rendu sur place, personne ne lui ouvre. Ricardo décide de rentrer par le jardin pour aller frapper à la porte d'entrée. Mais alors qu'il insiste, des policiers arrivent. Il tente de s'échapper, mais en vain. Les policiers lui demandent s'il voulait voler, en précisant qu'ils avaient été appelés par un monsieur qui l'avait vu enjamber l'enclos. Ricardo leur explique la situation, sans rentrer dans les détails de sa situation administrative. Au commissariat, où il est conduit pour un contrôle, on découvre que son passeport est faux. Les policiers vont lui demander de quitter le terri-

18 Elle était rentrée avec un visa touristique qui avait expiré au bout de trois mois. Elle entre en situation d'irrégularité, elle séjourne et travaille à Gênes pendant un an et demi et puis elle est régularisée grâce à la dernière « sanatoria » en 2002.

19 Ricardo m'explique que les connaissances de sa cousine sont surtout des filles sud-américaines qui travaillent dans l'aide à domicile et qui fréquentent l'église. 
toire italien appliquant ainsi le $«$ foglio di via $»^{20}$.

Ricardo ne quitte pas l'Italie, mais il abandonne l'idée de retourner voir son ex-patron. Il a peur de sortir de chez sa cousine, mais en même temps, en restant chez elle, il craint de lui créer des ennuis.

Deux semaines après, il rencontre un ami de sa cousine qui lui présente un artisan italien, qui l'embauche comme manœuvre, d'abord ponctuellement puis de plus en plus régulièrement. Ricardo reste avec lui un an : il est payé régulièrement et commence à s'acquitter de ses dettes (la maison de sa mère au Pérou avait été hypothéquée, sa cousine lui avait payé une partie du billet d'avion). Au bout d'un an, Ricardo demande à son patron de l'aider à obtenir un permis de séjour dans le cadre du « décret flux $2006 »^{21}$ afin de rentrer dans les quotas de main-d'œuvre étrangère que l'Italie établit chaque année par secteur d'activité. Son patron se dit disponible, mais quand Ricardo lui apporte les formulaires à compléter, le chantier arrive à son terme et son patron lui explique que la régularisation de sa position lui coûterait très cher $^{22}$ et qu'il ne peut pas se permettre de le garder. Ricardo se retrouve encore une fois sans travail et sans espoir de voir sa situation se régulariser. Après un mois et demi, il retrouve du travail par l'intermédiaire d'un compatriote maçon qui le fait rentrer dans une entreprise de huit salariés (plus quatre ou cinq ouvriers qui sont embauchés « à la journée »), « tous des Latinos », comme il me fait remarquer. Ricardo est le seul sans-papier.

$\mathrm{Au}$ moment de l'enquête, il travaille pour la même entreprise, mais toujours au noir. Le patron lui doit trois mois de salaire. Sous-payé par rapport aux autres salariés, les samedis et les dimanches lui sont rétribués comme des jours ouvrés, Ricardo pense que le patron n'a pas l'intention de demander sa régularisation lors de la publication du prochain " décret flux » : « je lui ai demandé de me payer les trois derniers mois de salaire, mais il m'a dit que si je sais pas attendre je peux m'en aller [...], le patron... pour lui l'important est de se faire le plus de sous possible, il voit pas l'intérêt de faire mes papiers... je lui ai même proposé de me payer moi-même la sécurité sociale et tout ça ! ». Ricardo est conscient d'occuper la place de l'« outsider utile » : « je compte pour rien... combien de fois je pouvais tomber du toit parce que c'est moi qu'ils envoient là-haut sans protection?».

20 Le « Foglio di via » est une mesure qui permet à l'autorité judiciaire d'imposer l'éloignement du territoire d'une personne suspectée d'activités illicites. Après la réforme de la loi sur l'immigration de 1998, l'étranger qui séjourne irrégulièrement en Italie a quinze jours pour quitter le territoire à partir de la date de l'émission du « foglio di via ». Actuellement, le " ddl-sicurezza » (décret de loi sur la sécurité), approuvé le 2 juillet 2009 par le Sénat malgré les contestations des associations de défense des droits de l'homme et des étrangers, de l'Église catholique et de l'opposition politique (faible), durcit les mesures contre l'immigration clandestine et irrégulière en introduisant des sanctions pénales pour l'immigré et pour toute personne susceptible d'avoir favorisé son séjour.

21 Le décret-flux (« decreto flussi ») renvoie à la politique de quotas qui établit chaque année le nombre de permis de séjour à délivrer par l'État italien. Ce système oblige entrepreneurs et travailleurs à faire semblant de ne pas se connaître lors du processus de recrutement car le décret ne s'adresse pas aux étrangers séjournant déjà sur le territoire de manière irrégulière.

22 La loi Bossi-Fini de 2002 et ses successives modifications prévoient un ensemble de contraintes parfois décourageantes pour l'employeur vis-à-vis de son salarié non-communautaire, dans le domaine du logement et des garanties financières à apporter pour l'éventuel rapatriement.

REMI 2011 (27) 2 pp. 35-55 
Le parcours de Ricardo est un échec, particulièrement sur le plan professionnel. Dans sa situation, là encore, le contexte politico-administratif et les marges de manœuvre des acteurs locaux du marché du travail sont déterminants, en réduisant notamment les possibilités de travail et de résidence légale.

\section{L'ACTION PUBLIQUE ET L'INSERTION DES IMMIGRÉS : CE QUE NOUS APPRENNENT LES QUATRE PARCOURS}

Les instruments de l'action publique et les configurations d'acteurs composent un cadre sociétal dans lequel l'immigré se déplace, négocie, s'insère ou est exclu, met en place des stratégies de promotion et/ou de survie selon la possibilité d'accéder à un statut reconnu.

Les parcours que nous avons reconstruits montrent comment certaines opportunités ou obligations se sont présentées suite à l'immersion de l'individu dans un contexte institutionnel, économique et politique. Ce dernier a défini un ensemble de règles et de contraintes qui a parfois favorisé l'émergence de liens sociaux qui lient l'immigré à son entourage (amis, collègues, famille, membres de l'administration, d'associations, de syndicats, etc.).

Ces deux dimensions - contextuelle et biographique - s'entrecroisent constamment dans le récit de nos interviewés ; d'ailleurs l'accès à un statut reconnu (titulaire d'un permis de séjour, d'un contrat en règle, stagiaire de la formation professionnelle, etc.) passe souvent par la médiation, obligatoire ou fortuite, d'un membre des réseaux dans lequel la personne s'inscrit (Bessin, Bidart et Grossetti, 2010).

L'intérêt de ces quatre parcours réside surtout dans leur capacité à mettre en relief le rôle des politiques publiques et des diverses configurations d'acteurs sur le territoire. La région Ligurie et la région PACA présentent sans aucun doute des similitudes quant aux besoins en main-d'œuvre de la branche et à la présence significative de travailleurs immigrés. Toutefois, les modalités de gestion de cette main-d'œuvre sont mises en place différemment par l'intermédiaire de configurations d'acteurs spécifiques.

Dans le cas d'Ali, l'employeur joue un rôle clé, et il ne s'agit évidemment pas d'un simple hasard. En France à cette époque, face à un contexte politique de fermeture officielle des frontières, des réseaux personnels de "solidarité intéressée " (le patron, en embauchant et fidélisant un bon travailleur, y trouvait aussi son compte) pouvaient se créer en vue de débloquer une situation administrative difficile, qui aurait empêché l'immigré étranger d'avoir accès à d'autres ressources (par exemple, la liberté de circulation ou la protection sociale) octroyées aux titulaires d'un statut reconnu, en l'occurrence la détention d'un permis de séjour. Mais ce processus est également le miroir d'un contexte socio-politique où le passage d'un statut de manœuvre au noir à celui de maçon déclaré pouvait se faire relativement facilement : la conjoncture économique était plutôt favorable pour le secteur ; dès lors, même si l'immigration économique était formellement suspendue depuis 1974, les formes de régulation territoriale du marché du travail permettaient aux entrepreneurs de favoriser la régularisation de travailleurs étrangers par 
l'emploi sans que la démarche ait été trop coûteuse en termes de temps ou de ressources pour les deux parties.

Le cas d'Abdel atteste de l'évolution du contexte politico-économique français. Son statut originel d'étudiant est destiné à « expirer », car un critère d'âge intervient dans la régulation de l'accès à un certain nombre de services spécifiques, comme le logement étudiant. Lorsqu'il parle de sa période « étudiante », Abdel la définit comme " normale » et en cela, il laisse apparaître un parcours «type » forgé par l'action publique : ce qui donne à penser à Abdel qu'il est en position d'être un étudiant « comme les autres », est le fait d'avoir moins de vingt-huit ans, de remplir les conditions d'éligibilité à l'accession à une chambre en cité universitaire, d'être inscrit à l'université, de travailler de temps en temps au noir dans la restauration (ce qui est perçu comme un boulot de « jeune »). En région Provence-Alpes-Côte d'Azur, l'action publique est sectorielle, et chaque catégoriecible est rendue opératoire afin d'intervenir sur un problème social spécifique (en l'espèce, le logement de jeunes étudiants). Restent problématiques des parcours non-linéaires qui tentent de transgresser les cloisonnements des institutions publiques sectorisées : c'est notamment le cas d'individus qui entreprennent de passer d'un statut d'étudiant étranger à celui de travailleur étranger en règle.

En l'occurrence, si la «gestion des parcours d'insertion des jeunes » est une des priorités affichées du conseil régional, la non-prise en compte des déphasages possibles entre les temporalités du parcours d'insertion d'un jeune immigré étranger et celles des contraintes administratives liées à son séjour est manifeste. La difficulté majeure qui en résulte tient à la tension entre deux temporalités a priori distinctes : celle d'une action publique sectorisée et celle de la trajectoire sociale de l'individu migrant. Le décalage entre le temps « linéaire ", qui fait référence à une notion de «parcours » telle qu'elle est construite et utilisée par les politiques de l'emploi, et le temps de l'immigré, souvent contraint d'osciller entre différents statuts (régulier-irrégulier, étudiant-salarié, résidentmobile, etc.), fait de la régulation des transitions sur le marché du travail des immigrés étrangers un non-objectif.

Les réseaux de soutien informels prennent alors la place de services publics absents ou corporatistes, donc, par définition, renvoyant à une " fermeture " (Barbier, 2008) : dans le cas d'Abdel, le réseau d'amis lui apporte des solutions dans l'urgence dans plusieurs domaines, comme dans le cas de son inscription à l'université, du logement et du travail.

Abdel met en place également ce que l'on pourrait appeler une version « soft » d'une « stratégie matrimoniale » qui, en perspective, pourrait correspondre à une protection supplémentaire : il propose à plusieurs reprises à sa copine de conclure avec lui un Pacte Civil de Solidarité (PaCS) ; pour Abdel c'est une tentative de se protéger de discriminations potentielles et de mettre toutes les chances de son côté afin de trouver « un vrai travail ». Abdel est un hybride : il n'est plus un "vrai étudiant », et pourtant il n'a pas eu accès à un statut substitutif en mesure de lui garantir l'accès à un autre panier de droits, notamment ceux réservés aux travailleurs. Il sollicite alors un système de solidarité informel et « de proximité », sans pouvoir résoudre durablement ses problèmes. 
Le récit de Karim met clairement en évidence le rôle joué par une institution territoriale : l'École du bâtiment. Dans le contexte italien, cet organisme est une structure de formation qui remplit de manière informelle les fonctions d'un centre de placement. Un réseau de professionnels et d'employeurs est particulièrement impliqué dans la construction de la relation formation-emploi. Une différence primordiale émerge donc par rapport au parcours précédent : dans le cas d'Abdel, le réseau mobilisé est informel, et a-professionnel ; de ce fait, il est en mesure de lui octroyer uniquement des possibilités de travail non-déclaré, que ce soit dans la restauration ou dans le bâtiment. Le système italien est plus poreux, plus bienveillant vis-à-vis de toute sorte de bricolage et d'interprétation de la législation. C'est au cas pas cas que les agents locaux, comme la secrétaire de l'École du bâtiment ou le fonctionnaire de la préfecture de Gênes, peuvent créer (ou annuler) des possibilités d'insertion là où d'autres ne verraient que des impasses probables. Dans le cas ligurien, les acteurs et les gatekeepers interagissent dans une configuration caractérisée par la territorialisation des organismes de branche (Lendaro, 2010), comme l'École du bâtiment ; aussi elle peut se permettre d'adopter des modes de fonctionnement propres, en mesure de prendre en considération la situation spécifique des immigrés du secteur. L'adoption d'une catégorie indigène d'immigré " en voie de régularisation » permet aux acteurs locaux d'inventer des solutions informelles face à des impasses d'ordre administratif, qui renvoient aux catégories rigides établies par la jurisprudence nationale qui peut ainsi être contournée. Des arrangements sont possibles et ils se révèlent encore plus déterminants dans le cas ligurien, puisque ces organismes jouent un rôle de médiateurs vers l'emploi beaucoup plus efficaces que les institutions publiques créées à cette fin, comme les centres pour l'emploi ${ }^{23}$. Ces organismes paritaires de branche, ainsi que d'autres acteurs du privé social comme les syndicats et les paroisses, sont les détenteurs d'informations et de contacts essentiels pour l'immigré, régulier et surtout irrégulier, à la recherche d'un travail.

Le cas de Ricardo est en ce sens emblématique : c'est grâce à sa fréquentation d'une paroisse génoise qu'il commence à tisser des liens avec d'autres immigrés, dont plusieurs sans-papier comme lui, qui se révéleront précieux du point de vue de ses premières expériences professionnelles dans le bâtiment. Ce parcours révèle à la fois l'absence de politiques publiques capables de réguler l'insertion sur le marché du travail, notamment dans les secteurs peu qualifiés comme le bâtiment et l'aide à domicile, et le pragmatisme de la société civile qui souvent devance les pouvoirs publics dans la mise sur pied de services de base (dortoirs ou accès aux soins), tout en proposant, de facto, de modifier les frontières entre éligibles et non-éligibles. L'Église devient une sorte d'agence pour l'emploi des immigrés sans papiers, qui attire naturellement des réseaux d'étrangers catholiques, comme les Sud-Américains. Le rôle de ces gatekeepers du privé social est encore plus important dans un contexte politique qui tente de gérer le phénomène migratoire en adoptant une posture « de l'urgence » : la mesure politique adoptée le plus régulièrement dans les vingt dernières années afin de réguler la présence immigrée sur le territoire a été la « sanatoria », c'est-à-dire la régularisation des immigrés séjournant irrégulièrement sur le territoire. La présence d'un nombre important d'immigrés irréguliers, due à des raisons multiples qui vont de l'accès relativement facile au marché du travail informel à la

23 Il s'agit des « centri per l'impiego », l'équivalent des agences locales pour l'emploi, qui ont une responsabilité départementale, sans qu'ils soient spécialisés, comme en France, dans un secteur professionnel. 
perméabilité des frontières italiennes, a donc catalysé l'intervention spontanée des acteurs publics et surtout privés du territoire, qui essayent de trouver des solutions à leur façon, en bricolant avec l'interprétation de directives et de décrets. Le « discretionnalisme » des acteurs locaux (que ce soit à la préfecture ou au secrétariat de l'École du bâtiment) participe également à la définition de contextes à géométrie variable dans lesquels l'immigré essaye de s'insérer, en ouvrant ou en réduisant l'horizon de ses possibilités.

Mais le parcours de Ricardo nous montre également tous les paradoxes du système législatif italien, qui actuellement repose sur les dispositions de la loi dite « BossiFini » adoptée en $2002^{24}$. La régularisation par l'emploi qu'Ali a vécue il y a vingt ans en France prend, à l'heure actuelle, les contours d'un parcours du combattant dans le contexte italien : la procédure est avant tout très coûteuse pour l'employeur, en termes de garanties à assurer concernant les ressources financières du salarié pour le paiement du loyer, des titres de transport pour le retour au pays, etc. (Tursi, 2005). Les conséquences les plus visibles sont le désintérêt, voire la méfiance, des entrepreneurs vis-à-vis de ces procédures et le renforcement de niches de travail non déclaré, qui est peu sanctionné et plus rentable du point de vue des employeurs. Ainsi, le parcours de Ricardo est une impasse, où le temps d'attente d'une régularisation se prolonge, où le travail non déclaré est la seule option, où le travailleur n'est pas protégé en cas d'accident de travail en étant fragilisé vis-à-vis du patron et des autres salariés déclarés.

\section{EN GUISE DE CONCLUSION}

Ce travail a voulu questionner l'action publique et son influence sur les parcours d'insertion via la reconstruction des transitions et des événements-clés qui émergent des récits des immigrés et surtout à travers les liens entre le contexte institutionnel et les choix possibles, les opportunités, les risques individuels.

Le statut, et la catégorie-cible d'une politique publique ont un double pouvoir : d'abord de distinguer, de faire le tri entre « éligibles » et « exclus » ou « non-concernés », et de définir les caractéristiques des premiers ; dans un même temps, ils permettent à l'immigré de se positionner par rapport « aux autres », de se percevoir ou pas comme étant « comme les autres » du point de vue des droits et des devoirs.

Les exemples examinés ici montrent que les configurations d'acteurs sur les deux territoires ne sont pas identiques et que des formes d'arrangement sociétales émergent, donnant lieu à des modalités d'insertion professionnelle qui, sans être figées, sont plus typiques d'un contexte à l'autre. Ainsi, dans les deux régions, des enjeux similaires concernant l'insertion professionnelle des immigrés dans une branche caractérisée par des problématiques voisines (comme la difficulté formelle à fidéliser la main-d'œuvre jeune) n'induisent pas les mêmes catégorisations ni les mêmes configurations d'acteurs, car la construction de problèmes collectifs suit des cheminements différents, tout comme les parcours d'insertion des immigrés.

Ces parcours témoignent également d'un effet historique important qui met

24 Loi $^{\circ} 189 / 2002$. 
en évidence le caractère évolutif de ces configurations d'acteurs et du contexte institutionnel lié aux politiques publiques : les événements clés des parcours d'insertion, comme l'accès à un emploi déclaré et éventuellement durable ou au statut d'immigré régulier, se déroulent dans une phase spécifique de l'histoire migratoire des deux pays. Les deux parcours d'immigration plus récents sont plus problématiques, du moment où l'accès au statut d'immigré régulier pour raisons économiques est plus difficile à obtenir et reflète les logiques à la base des législations actuelles sur l'immigration qui renvoient à la nécessité de réduire et contrôler le phénomène et de sélectionner la main-d'œuvre étrangère à partir de critères d'utilité économique.

Notre point de départ selon lequel l'action publique, par ses opérations de ciblage, est capable d'orienter les parcours de vie, nous a conduit à réfléchir aux contextes institutionnels et aux configurations d'acteurs publics et privés dans lesquels les cheminements d'insertion des immigrés se déroulent. Les quatre parcours nous ont montré, à des degrés différents, qu'en l'absence de politiques universalistes et dans des contextes où la régulation du marché du travail se fait davantage par le biais de politiques sectorielles, parfois « excluantes » ou qui « laissent-faire », des possibilités d'échapper aux contraintes institutionnelles existent, notamment grâce aux réseaux des marchés locaux (Sassen, 1995).

\section{Références bibliographiques}

AMBROSINI Maurizio (2008) Surprises italiennes : une étrange continuité dans la gestion de l'immigration, Journée d'Étude « Immigration et Marchés du Travail », 8 avril 2008, Maison Méditerranéenne des Sciences de l'Homme, Aix en Provence.

AMBROSINI Maurizio (2005) Sociologia delle migrazioni, Bologna, Il Mulino, 292 p.

AMBROSINI Maurizio (2001) La fatica di integrarsi. Immigrazione e lavoro in Italia, Bologna, Il Mulino, $216 \mathrm{p}$.

BARBAGLI Marzio, COLOMBO Asher, SCIORTINO Giuseppe (2004) I sommersi e i sanati: le politiche di regolarizzazione degli immigrati in Italia, Bologna, Il Mulino, $280 \mathrm{p}$.

BARBIER Jean-Claude (2008) L'« activation » de la protection sociale, existe-t-il un modèle français?, in Anne-Marie Guillemard, Où va la protection sociale, Paris, PUF, pp. 165-182.

BEAUD Stéphane et PIALOUX Michel (1998) Notes de recherche sur les relations entre Français et immigrés dans l'usine et le quartier, Genèses, 30, pp. 101-121.

BÉHAR Daniel, ESTÈBE Philippe et EPSTEIN Renaud (1998) Les détours de l'égalité. Remarques sur la territorialisation des politiques sociales en France, Revue Française des Affaires Sociales, 54 (4), pp. 81-94.

BESSIN Marc, BIDART Claire et GROSSETTI Michel (2010) Bifurcations. Les sciences sociales face aux ruptures et à l'événement, Paris, La Découverte, $400 \mathrm{p}$.

CASTEL Robert (1995) Les métamorphoses de la question sociale, Paris, Fayard, 813 p.

DE RUDDER Véronique, POIRET Christian et VOURC'H François (2000) L'inégalité raciste. L'universalité républicaine à l'épreuve, PUF, Paris, $224 \mathrm{p}$.

DOYTCHEVA Milena (2007), Une discrimination positive à la française ? Ethnicité et territoire dans les politiques de la ville, Paris, La Découverte, $228 \mathrm{p}$.

DEMAZIÈRE Didier et DUBAR Claude (1997) Analyser les entretiens biographiques : l'exemple de récits d'insertion, Paris, Nathan, $330 \mathrm{p}$.

DUBAR Claude (2001) La construction sociale de l'insertion professionnelle, Éducation et Société, 7 (1), pp. 23-36. 
FELLINI Ivana, FERRO Anna and FULLIN Giovanna (2007) Recruitment processes and labour mobility: the construction industry in Europe, Work, employment and society, 21 (2), pp. 277-298.

GIRAUD Olivier (2004) La comparaison comme opération de réduction de la complexité. Le cas de la formation professionnelle, Revue Internationale de Politique Comparée, 11 (3), pp. 349-368.

GIRAUD Olivier et LUCAS Barbara (2007) Jeux d'échelle et référentiels dans les politiques du handicap en Suisse, in Alain Faure, Jean-Philippe Leresche, Pierre Muller et Stéphane Nahrath Éds., Action publique et changements d'échelles : les nouvelles focales du politique, Paris, L'Harmattan, pp. 119-134.

GROSSETTI Michel (2006) L'imprévisibilité dans les parcours sociaux, Cahiers Internationaux de Sociologie, 120, pp. 5-28.

GUIRAUDON Virginie (2006) L'intégration des immigrés ou la politique de l'esquive. Réformer sans changer de modèle ?, in Bruno Palier, Peter A. Hall et Peter D. Culpepper Éds., La France en mutation 1980-2005, Paris, Presses de Science Po, pp. 265-301.

IVE Graham J. and GRUNEBERG Stephen L. (2000) The Economics of the Modern Construction Sector, London, Macmillan Press, 304 p.

JOBERT Annette (Éd.) (2008) Les nouveaux cadres du dialogue social. Europe et territoires, Bruxelles, P.I.E. Peter Lang, 267 p.

JOUNIN Nicolas (2008) Chantier interdit au public, Paris, La Découverte, 276 p.

LALLEMENT Michel et SPURK Jan (2003) Stratégies de la comparaison internationale, Paris, CNRS Editions, $384 \mathrm{p}$.

LASCOUMES Pierre et LE GALES Patrick (Éds.) (2005) Gouverner par les instruments, Paris, Presses de la Fondation Nationale des Sciences Politiques, $371 \mathrm{p}$.

LECLERC-OLIVE Michèle (1997) Le dire de l'évènement (biographique), Lille, Presses Universitaires du Septentrion, $260 \mathrm{p}$.

LENDARO Annalisa (2010) L'État et les « autres ». Visibilisation de la main-d'œuvre immigrée en PACA (FR) et en Ligurie (ITA), in Lionel Jacquot, Jean-Pascal Higelé, Hervé Lhotel et Christophe Nosbonne Coord., Formes et structures du salariat : crise, mutation, devenir, tome I : De la construction sociale du rapport salarial, Nancy, PUN.

LINHART Robert (1978) L'établi, Paris, Les Editions de Minuit, 180 p.

MACHKOVA Elena (2009) Risques de travail, risques de l'emploi. L'exemple des travailleurs intérimaires du secteur du bâtiment, thèse en sociologie, Université Paris V, CEMS-EHESS.

MAURICE Marc, SELLIER François et SILVESTRE Jean-Jacques (1982) Politique d'éducation et organisation industrielle en France et en Allemagne : essai d'analyse sociétale, Paris, PUF, $382 \mathrm{p}$.

MAYER Karl Ulrich and SCHOEPFLING Urs (1989) The State and the Life Course, Annual Review of Sociology, 15, pp. 187-209.

PIORE Michael (1979) Birds of Passage. Migrant Labour and Industrial Societies, Cambridge, Cambridge University Press, $229 \mathrm{p}$.

SASSEN Saskia (1995) Immigration and Local Labor Markets, in Alejandro Portes Ed., The Economic Sociology of Immigration. Essays on Networks, Ethnicity, and Entrepreneurship, New York, Russel Sage Foundation, 310 p.

TRIPIER Maryse (2004) L'immigré, analyseur de la société, Terrains \& Travaux, 2 (7), pp. 173-185. TRIPIER Maryse (1990) L'immigration dans la classe ouvrière en France, Paris, L'Harmattan, 332 p.

TURSI Armando (Ed.) (2005) Lavoro e immigrazione. Commento alle norme della legge n. 189 del 2002, Torino, Giappichelli, 516 p.

VERDIER Eric (2000) Reintroducing public action into societal analysis, in Marc Maurice and Arndt Sorge Eds., Embedding organizations: societal analysis of actors, organizations and socio-economic context, Amsterdam, John Benjamins publishing company, pp. 325-338.

VIET Vincent (1998) La France immigrée. Construction d'une politique. 1914-1997, Paris, Fayard, 510 p. ZINCONE Giovanna and CAPONIO Tiziana (2004) Immigrant and immigration policy-making. The case of Italy, country report, $1^{\text {ère }}$ Conférence Générale IMISCOE, Coimbra, 3-4 décembre 2004. 


\section{Le pouvoir de la catégorie. Les politiques publiques et l'insertion professionnelle des immigrés en France et en Italie}

\section{Annalisa Lendaro}

L'article propose une analyse de l'action publique via la reconstruction de parcours de vie et de transitions concernant l'insertion professionnelle et l'évolution de carrière des immigrés : l'intérêt est de mesurer l'influence des politiques d'emploi et d'immigration en termes d'opportunités d'accès à l'emploi et aux droits sociaux. Le fondement de la politique d'immigration française a longtemps été une forme de républicanisme assimilationniste, qui sollicitait les immigrés pour qu'ils s'intègrent surtout à travers l'insertion sur le marché du travail. Les politiques publiques prennentelles en considération les besoins de l'individu au point de s'interroger sur les problématiques de l'étranger non-communautaire ? L'intérêt est celui d'une analyse critique des catégories-cibles, afin de mettre en évidence la mesure dans laquelle on explicite ou au contraire, on rend invisibles les populations en cause. Le détour par la situation italienne permet d'apporter un regard nouveau sur les politiques d'immigration françaises et sur la gestion de la main-d'œuvre immigrée à travers un exercice comparatif qui s'inspire de l'analyse sociétale « revisitée ».

\section{The Power of Categories. Public Employment Policies and Immigration in France and Italy}

\section{Annalisa Lendaro}

The paper explores public employment policies through life course analysis and focuses on transitions concerning the professional employment and careers of immigrants. The aim is to point out the influence of employment and immigration policies on the opportunities of immigrants in the areas of labour employment and the acquisition of social rights. French migratory policy has for a long time been based on a form of republican assimilation, i.e. immigrants are to be integrated via employment on the labour market. Do public policies consider the needs of one individual to the extent that they wonder about the specific problems of the non-European foreigner? The aim is to critically analyse the target categories to underline how it is possible to transform a population into a public priority, or conversely, to make it invisible. The Italian case enables us to look differently at the French immigration and employment systems: this comparative exercise draws its inspiration from a "revisited" societal analysis.

\section{El poder de las categorías. Políticas públicas e inserción laboral de inmigrantes en Francia y en Italia}

\section{Annalisa Lendaro}

Este artículo presenta un análisis de la acción pública a través de la reconstrucción de trayectorias de vida y de las transiciones ligadas a la inserción y evolución profesional de inmigrantes. Su objetivo es medir la influencia de las políticas laborales y migratorias en términos de oportunidades de acceso al trabajo y a los derechos sociales. Durante mucho tiempo, la política migratoria francesa estuvo fundada en una forma de republicanismo asimilacionista que limitaba la integración de inmigrantes a su inserción laboral. ¿Las políticas públicas toman en cuenta las necesidades de los individuos, es decir, se interesan en las problemáticas que afectan al inmigrante no europeo? El propósito de este artículo es lograr un análisis crítico de las categorías objetivo de estas políticas para mostrar en qué medida se destaca o se subestima la presencia de ciertos grupos. El caso italiano permite observar las políticas migratorias francesas sobre la gestión de la mano de obra migrante de forma comparativa; un enfoque inspirado en una reconsideración del análisis societal. 